\title{
IMPLEMENTATION OF LOW PASS FiLTERS WITH REAL OPAMPS: WHERE IS THE LIMIT?
}

\author{
Martin Pospisilik, Tomas Dulik, Milan Navratil \& Vaclav Mach
}
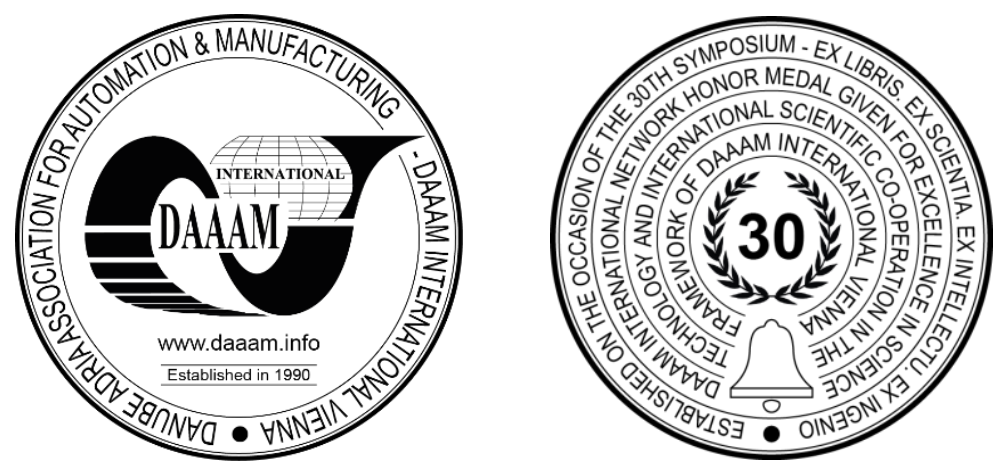

This Publication has to be referred as: Pospisilik, M[artin]; Dulik, T[omas]; Navratil, M[ilan] \& Mach, V[aclav] (2019). Implementation of Low Pass Filters with Real OpAmps: Where is the Limit?, Proceedings of the 30th DAAAM International Symposium, pp.0248-0253, B. Katalinic (Ed.), Published by DAAAM International, ISBN 978-3-90273422-8, ISSN 1726-9679, Vienna, Austria

DOI: $10.2507 / 30$ th.daaam.proceedings.032

\begin{abstract}
Active filters based on RC components together with operational amplifiers are quite common functional blocks to be met across different branches of electronics design. During the past, their topology has settled on several well-known and effective solutions. As an example, Sallen-Key or Multiple Feedback topology can be named. The designers can find a huge number of "cookbooks". According to them they can find help with designing their filters. However, the theory described in these cookbooks usually fits ideal operational amplifiers in a good manner, but, unfortunately, the real parameters of the operational amplifiers may spoil the real behaviour of the design significantly. This problem occurs especially at very high frequencies that are typical for injection of electromagnetic interferences into low frequency signal lines.

The authors of this paper have tested several antialiasing (low-pass) filter designs and provide results of measurement at real filters compared to the simulations. Moreover, they provide a discussion on why the results of the simulation do not fit the real behaviour of the filters and suggest improvements in the design of the low pass filters in that way so their utility value was improved.
\end{abstract}

Keywords: Low Pass Filters; Electromagnetic Compatibility; Interferences; Antialiasing; Sallen-Key; Multiple Feedback Filter.

\section{Introduction}

As modern electronics employs digital signal processing, there is a need for limiting of the bandwidth of the processed signal prior to its sampling. The filters used at the front-ends of A/D converters are called antialiasing filters and their main task is to suppress frequencies that cannot be processed due to limited frequency of the sampling process. Naturally, these filters are constructed as low pass filters (LPF) and, usually, are of higher orders. This is because steeper modulus of the transfer function enables broader effective bandwidth of the processed signal. In systems with A/D conversion, both digital and analogous signals usually meet at a single printed circuit board. Therefore, the suppression of frequencies above the defined limit must robust enough as there is always a risk of occurrence of broadband interferences. 
The purpose of the hereby described study is quite straightforward. In real application, it was found that the circuit constructed in accordance with the diagram depicted in the Figure 4 did not work properly. Ways to improve its performance were attempted to find. The question was how much the performance can be improved with a minor change in the circuit design.

\subsection{Implementation of OpAmps}

The modern LPFs are usually constructed as closed functional blocks employing operational amplifiers together with appropriate feedback network, defining the required transfer function. This topic is well described across many books and publications, for example [1], [2].

Among the most common constructions, LPFs using the Sallen-Key [3] or Multiple Feedback [4] topology can be found. The basic circuit configuration of 2 nd order LPF using these topologies is depicted in the Figure 1. Other examples can be found in [5].

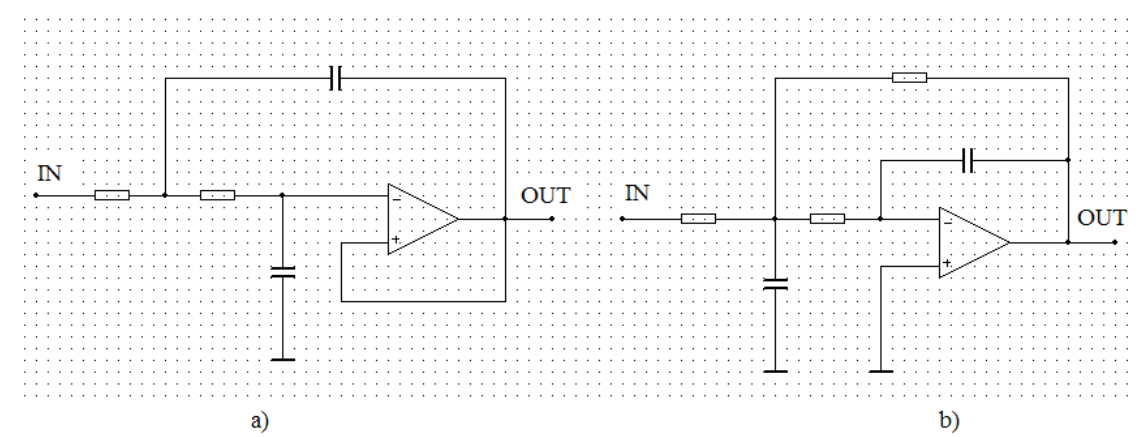

Fig. 1. Basic $2^{\text {nd }}$ order unity gain low pass filter using Sallen-Key (a) topology or Multiple feedback (b) topology

The values of the resistors and capacitors in the feedback network depend on the approximation function the designer chose. Usually, Butterworth or Chebyshev approximations [6] are employed, targeting on the maximum steepness of the transfer function's modulus.

\subsection{Transfer function measurement}

The transfer function of any circuit describes its modulus and phase, both depending on frequency. The measurement of the transfer function can be processed automatically by means of a vector network analyser. A suitable device to process measurements on low-frequency filters is Bode 100 by Omicron Lab, operating in the frequency range from $1 \mathrm{~Hz}$ to $50 \mathrm{MHz}$. This device is utilized at the Faculty of Applied informatics and was employed in measurements described within the framework of this paper. Utilization and port configuration of this analyser is described in the Figure 2 . The figure shows not only the typical wiring of the analyser, but also the analyser's configuration menu, enabling gain/phase measurements. This setting has been used for the purposes of the hereby described measurements.

\subsection{Non-ideal OpAmps}

Both circuits depicted in Figure 1 expect ideal OpAmps, the closed loop output impedance of whose is as low as zero. However, in reality, the output impedance of conventional OpAmps is usually quite high, and moreover, it rises accordingly with the frequency of the processed signal. This can be demonstrated by means of several figures from the datasheet of one of the common OpAmps, TL 07x, that can be found in the Figure 3. It is obvious, that at low frequencies, the output impedance of the OpAmp is approximately in hundreds of Ohms. Moreover, at frequencies above $100 \mathrm{kHz}$, the output peak-to-peak voltage is limited considerably as a consequence of a limited slew rate of the circuit. As a result of this, the feedback networks of the circuits depicted in the Figure 1 does not see a zero impedance at the outputs of the OpAmps, but, at high frequencies, the impedance at the output node of the OpAmps is considerably high, especially when compared to the impedance of the feedback capacitors, the capacitive reactance of whose decreases with the frequency. As a result of this, at very high frequencies the circuits depicted in the Figure 1 are "bypassed" by the feedback capacitors and above their critical frequency, they definitely do not behave as low pass circuits.

\section{Filter implementation in practice}

In the Figure 4 there is a circuit diagram of a typical $3^{\text {rd }}$ order anti-aliasing filter based on the Sallen-Key topology, using a TLV2442 OpAmp. The cut off frequency lies approximately at $16 \mathrm{kHz}$. The filter has been simulated by means 
of National Instruments Multisim software, using SPICE libraries for the circuit elements including the OpAmp. Together with the circuit diagram, a simulated modulus of the filter's transfer function is depicted.

The filter depicted in the Figure 4 was employed as a front-end of an A/D converter in a device for signal processing. Smooth attenuation of high frequencies was expected in order to fulfil the requirements of Nyquist-Shannon sampling theorem.

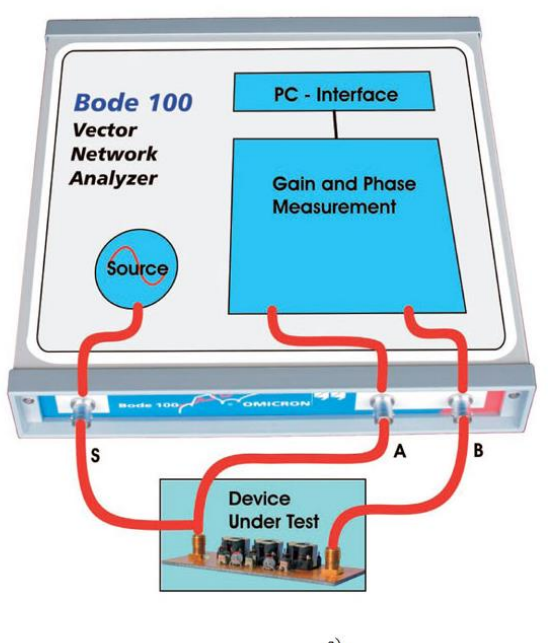

a)

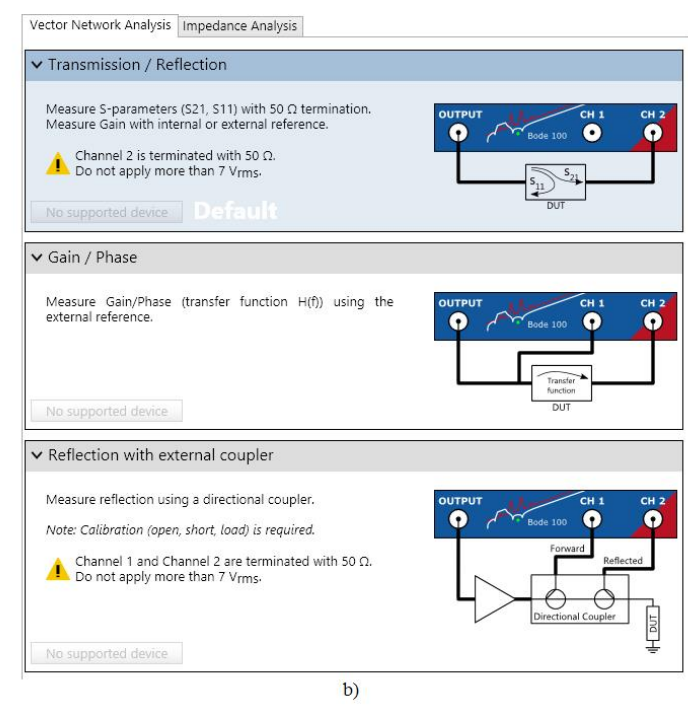

Fig. 2. Vector network analyser Bode 100 typical wiring (a) and setup screen (b)

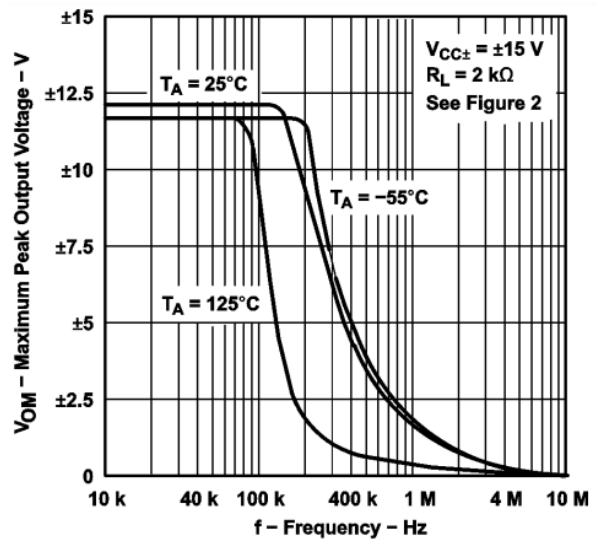

a)

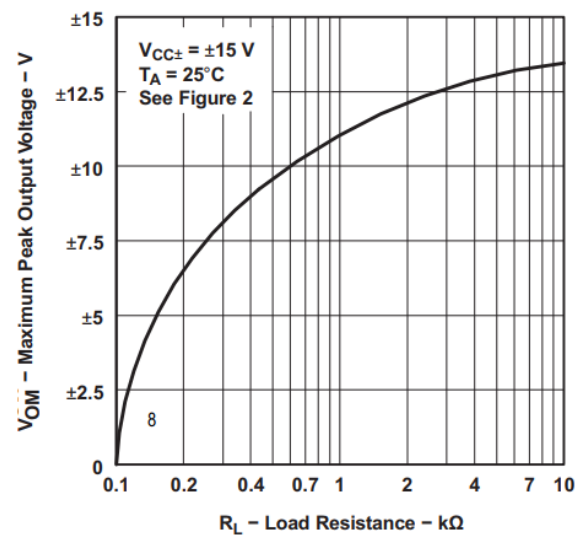

b)

Fig. 3. Typical performance of a conventional OpAmp TL07x, namely Maximum peak output voltage versus frequency (a) and Maximum peak output voltage versus load resistance [7]

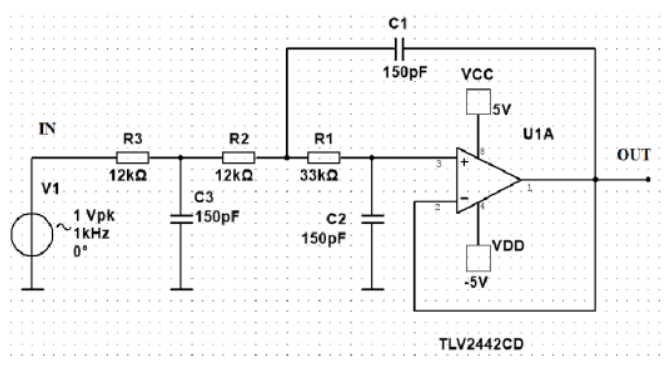

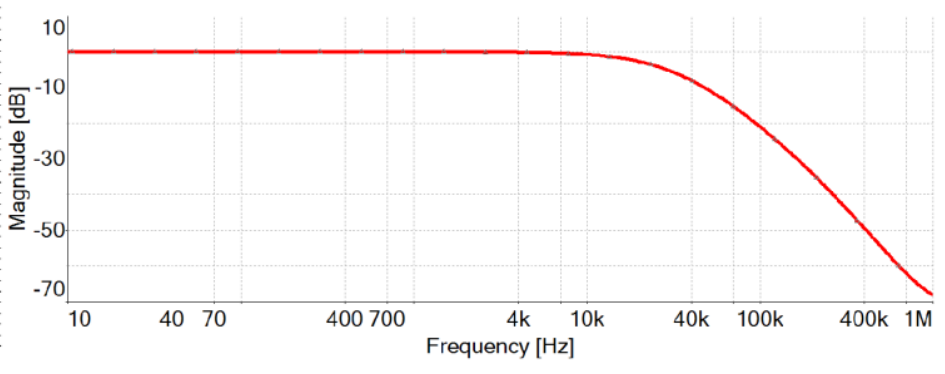

b)

Fig. 4. Simulated low-pass filter with Sallen-Key topology (a) and its transfer function's modulus obtained by Multisim software (b) 
However, the constructed device was tested by means of the vector network analyser Bode 100 and a serious finding was discovered. The SPICE model of the OpAmp did not respect the increase in the OpAmp's output node in any way. The measurement shown that the filter operates well at frequencies below approximately $125 \mathrm{kHz}$. At higher frequencies it failed quite significantly, as can be seen it the Figure 5 that shows data from the measurement in comparison to the simulated results.

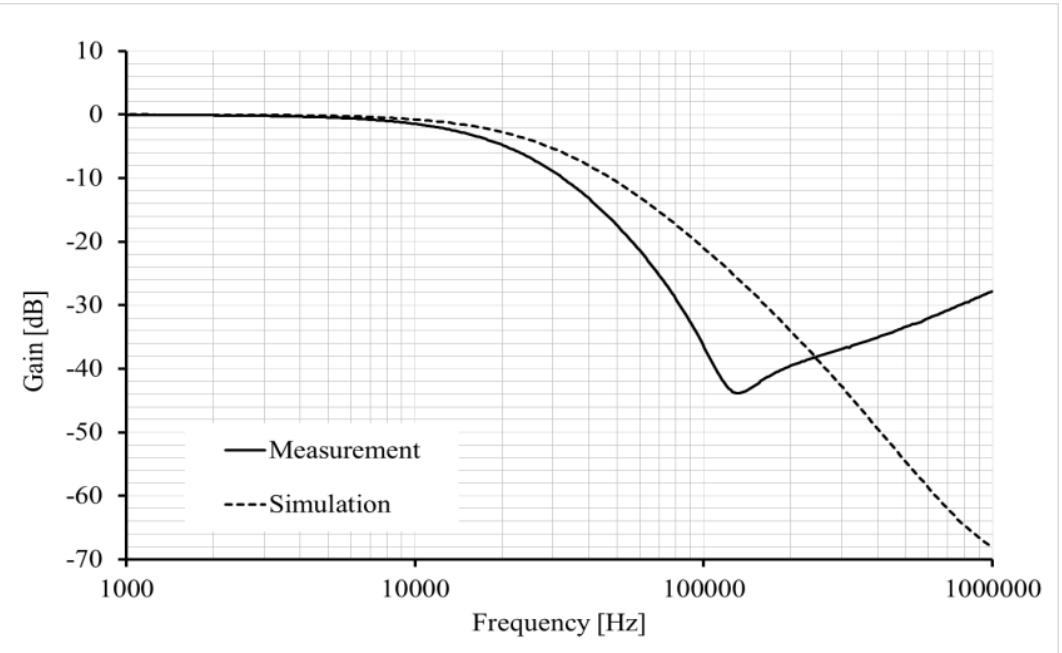

Fig. 5. Difference between simulated and measured results, circuit depicted in Fig. 4

\section{Improved construction}

According to simulation results depicted in the Figure 5 it is obvious that the anti-aliasing filter circuit depicted in Fig. 4 would not work properly at high frequencies. The simulation software tactfully concealed the increased output impedance of the OpAmp at high frequencies, resulting in crosstalk of the filtered signal between the input and the output of the filter through the feedback capacitor $\mathrm{C} 1$. As the device using this filter was intended to process mixed signals with a risk of conducted interferences at high frequencies, a better solution had to be introduced.

According to [8], the multiple feedback topology shows better performance at high frequencies. Moreover, it is expedient to move the first pole of the transfer function, created by the devices R3, C3 (see Figure 4) to the back-end of the OpAmp where it will work independently to the OpAmp's performance. Instead of the OpAmp type TLV2442, a bit faster TL 072 was employed (see [7]). The cut off frequency was decreased to $10 \mathrm{kHz}$. However, the experiment was made with both of the OpAmps and the differences between their performances was negligible.

A circuit diagram of the improved filter is depicted in the Figure 6. The performance of the filter in comparison with measured results is provided in the Figure 7.

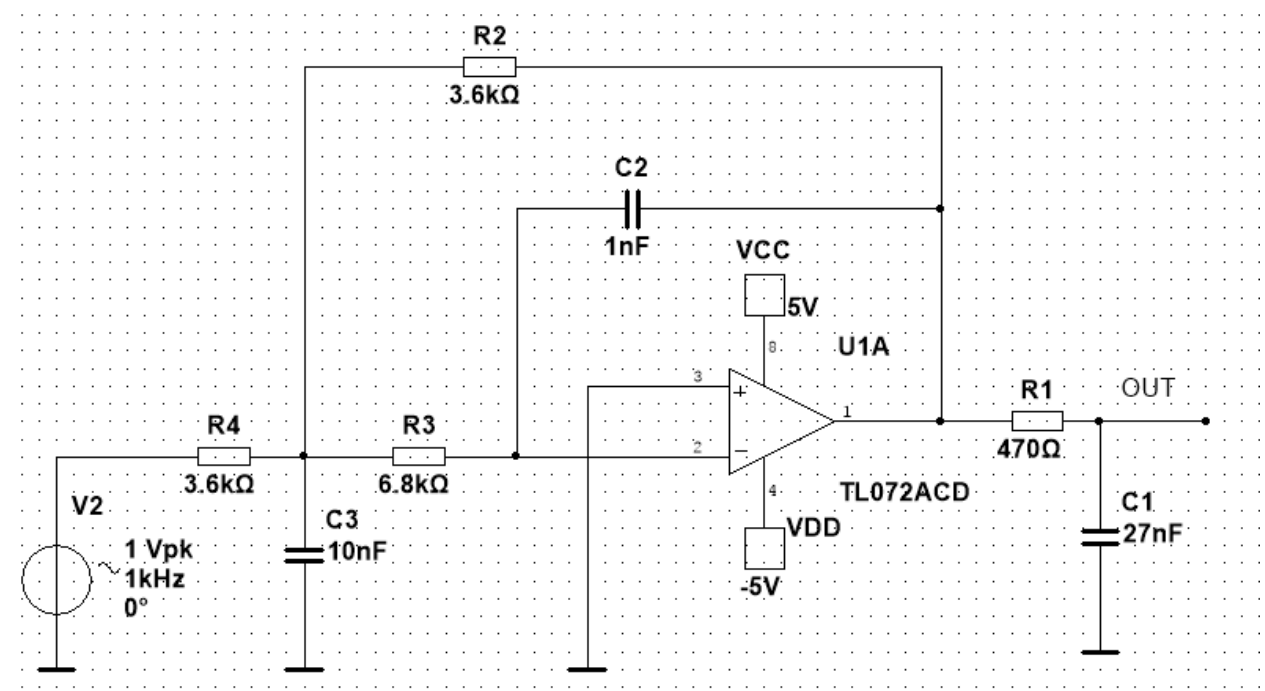

Fig. 6. Circuit diagram of the improved anti-aliasing filter 


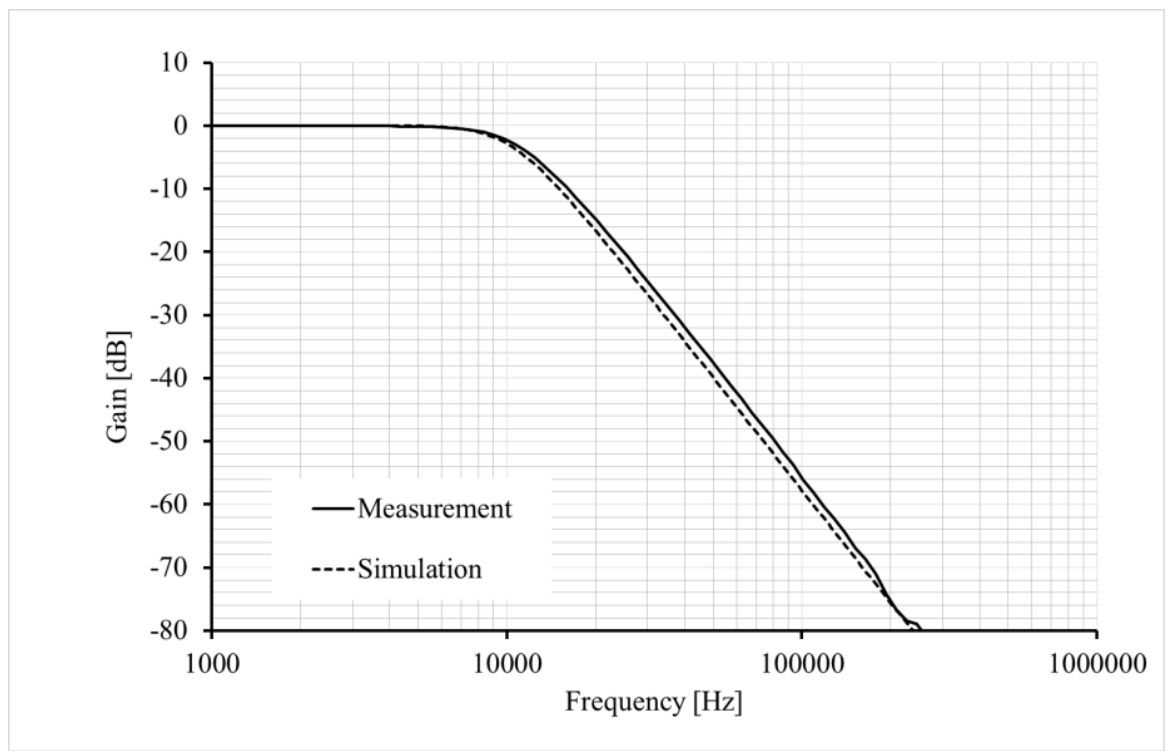

Fig. 7. The performance of the circuit depicted in Fig. 6. Difference between measurement and simulation

\section{Conclusions}

According to theoretical premises, both circuits (see Fig. 5 and Fig. 7) should provide similar transfer functions. However, it was found by experiment that the Multiple feedback topology (see Fig. 1b) is less sensitive to physical limitation of the OpAmps than the Sallen-Key topology (see Fig. 1a). Although the OpAmp TL072 is less expensive than TLV2442 and its parameters are not perfect (see Fig. 4), the performance of Multiple feedback structure was better than the performance of Sallen-Key structure with TLV2442. Although the circuit depicted in the Figure 5 reached attenuation no higher than $30 \mathrm{~dB}$ at the frequency of $1 \mathrm{MHz}$, the circuit depicted in the Figure 7 kept the attenuation below $80 \mathrm{~dB}$ at the same frequency. In order to prove the influence of the OpAmp type, experiments were made with both of them - with TLV2442 as well as with TL072. The difference between the two OpAmps was negligible, unlike the difference between the topologies, which was quite enormous.

The findings can be listed as follows:

- The simulation SW, although modern and sophisticated, may not simulate all parameters of the OpAmps correctly.

- At high frequencies, the performance of the filters is dependent on the circuit topology and it is advisable to take the output impedance of the OpAmp into account as when it is increased with the frequency, the whole filter becomes bypassed by the feedback network.

- When constructing anti-aliasing filters, high order low pass filters are preferred, allowing the constructer to reach wide bandwidth of the processed signal. However, these filters must be capable of suppression of non-wanted signals in a wide range as fast transients originated in digital circuits may reach the input of the A/D converter and cause aliased interferences in the processed signals.

Obviously, there are still limitations originating from a finite slew rate of the OpAmps and their other parasitic parameters. However, a minor design change from Sallen-Key to Multiple feedback topology helped us to enlarge the applicable frequency range of the circuit quite greatly.

In the future we plan to create a testbench for different low pass filter topologies with the possibility of changing OpAmps in a slot in order to study the performance of various OpAmps in different filter topologies.

\section{Acknowledgments}

This paper is supported by the Ministry of Education, Youth and Sports of the Czech Republic within the National Sustainability Programme project No. LO1303 (MSMT-7778/2014) and also by the Internal Grant Agency of Tomas Bata University under the project No. IGA/CebiaTech/2019/003. 


\section{References}

[1] Martinek, P.; Bores, P.; Hospodka, J. (2003). Electric Filters [Elektricke filtry], Czech Technical University Press, ISBN 80-01-02765-1, Prague

[2] Hajek, K.; Sedlacek, J. (2002). Frequency filters [Kmitoctove filtry], BEN, ISBN 80-7300-023-7, Prague

[3] http://www.ti.com/lit/an/sloa024b/sloa024b.pdf, Texas Instruments (2002). Analysis of the Sallen-Key Architecture, Published by Texas Instruments. Accessed on: 2019-10-02

[4] https://www.analog.com/media/en/training-seminars/tutorials/MT-218.pdf, Analog Devices (2012). Multiple Feedback Band-Pass Design Example, Published by Zumbahlen, H. Accessed on: 2019-10-02

[5] Dostalek, P[etr]; Vasek, V[ladimir] \& Dolinay, J[an] (2018). Microcontroller Based Control System with Flexible Configuration, Proceedings of the 29th DAAAM International Symposium, pp.0215-0224, B. Katalinic (Ed.), Published by DAAAM International, ISBN 978-3-902734-20-4, ISSN 1726-9679, Vienna, Austria, DOI: 10.2507/29th.daaam.proceedings.031

[6] Mudde, M. H. (2017). Chebyschev approximation. University of Groningen, Faculty of science and engineering. Groningen

[7] http://www.ben.cz/_d/datasheet/t1072.pdf, TL 072 datasheet by ST Microelentronics, Accessed on: 2019-10-05.

[8] Steffes, M. (2006). Design Methodology for MFB Filters in ADC Interface Applications. Application report published by Teaxas Instruments, Dallas. 